\title{
Macrophage polarisation changes within the time between diagnostic biopsy and tumour resection in oral squamous cell carcinomas an immunohistochemical study
}

\author{
M Weber ${ }^{\star}, 1, \mathrm{P}$ Moebius ${ }^{1}$, M Büttner-Herold ${ }^{2}, \mathrm{~K}$ Amann $^{2}$, R Preidl ${ }^{1}, \mathrm{~F}$ W Neukam ${ }^{1}$ and F Wehrhan ${ }^{1}$ \\ ${ }^{1}$ Department of Oral and Maxillofacial Surgery, Friedrich-Alexander University Erlangen-Nürnberg, Erlangen, Germany and \\ ${ }^{2}$ Department of Nephropathology, Institute of Pathology, Friedrich-Alexander University Erlangen-Nürnberg, Erlangen, \\ Germany
}

Background: The prognosis of solid malignancies has been shown to depend on immunological parameters, such as macrophage polarisation (M1/M2). Recently, it was reported that preoperative oral surgery leads to a worsening of oral squamous cell carcinomas (OSCC) prognosis. Diagnostic incision biopsies are oral surgery procedures that might lead to healing-associated M2 macrophage polarisation with a potential negative influence on tumour biology. No studies have compared macrophage polarisation in OSCC biopsies and tumour specimens.

Methods: Preoperative diagnostic incision biopsies $(n=25)$ and tumour resection specimens $(n=34)$ of T1/T2 OSCC were processed for immunohistochemistry to detect CD68-, CD11C-, CD163- and MRC1-positive cells. Samples were digitised using whole-slide imaging, and the expression of macrophage markers was quantitatively analysed.

Results: Carcinoma tissues obtained during OSCC tumour resections showed a significantly $(P<0.05)$ increased CD163 cell count (M2 macrophages) compared with tissues obtained during preoperative incision biopsies. Additionally, the CD163/CD68 ratio (an indicator of M2 polarisation) was significantly $(P<0.05)$ higher in tumour resection specimens than in biopsies.

Conclusions: This study revealed for the first time an increase in M2 polarisation in samples obtained during OSCC tumour resection surgery compared with preoperative incision biopsies. The biopsy-induced tissue trauma might explain the observed shift in macrophage polarisation towards the tumour-promoting M2 type and could lead to accelerated tumour progression.

Although there have been advances in multimodal treatment options for oral squamous cell carcinoma (OSCC) in the past 20 years (Pignon et al, 2009), the overall prognosis of this specific cancer type has not improved as substantially as for other malignancies (Kaatsch et al, 2013). This observation serves as a call to action to critically analyse the current diagnostic and therapeutic regime by considering all relevant biological tumour characteristics.
Prognostic assessment and therapeutic decision-making depend on TNM staging and histological grading. The preoperative staging process includes radiological imaging and diagnostic incision biopsies. Small OSCC (T1 and T2) are commonly treated by surgical tumour resection (Wolff et al, 2012).

For these reasons, OSCC patients are commonly subjected to two invasive surgical procedures (the diagnostic incision biopsy 
and the later tumour resection) with a time interval of some days or weeks between the two procedures (Wolff et al, 2012).

Oral surgery, such as diagnostic incision biopsies, represents local tissue trauma leading to wound-healing responses. A recent study showed that OSCC patients undergoing preoperative oral surgery exhibited a significant increase in lymph node metastases $(\mathrm{pN}+)$ and distant metastases (cM1) and a significantly decreased 5 -year survival rate (Takahashi et al, 2013) when compared with patients who did not undergo preoperative oral surgery. This report and other studies (Ohtake et al, 1990; Suzuki et al, 1998) suggested a correlation between local tissue trauma and increased malignancy in oral cancer.

These suggestions are not new; a possible negative effect of tumour biopsies has been discussed in the literature for years. A report from 1978 postulated that tumour surgery had biological consequences and even that the surgical procedures themselves could provoke the exacerbation of metastases (Krokowski, 1979). Another old study stated that cytoreductive surgery in testicular cancer was followed by a dramatic exacerbation of the disease in some cases (Lange et al, 1980).

Several further reports in the older literature showed a negative influence of surgical intervention on tumour progression (Keller, 1983; Bogden et al, 1997), but no biological explanations for the observed effects were given.

Recent findings from an animal study supported these suggestions. Biopsies were shown to significantly increase the frequency of lung metastases by 3-5-fold (Hobson et al, 2013). In addition, a significant increase in primary tumour growth was observed in mice that underwent biopsies (Hobson et al, 2013). Immunohistochemically, an increased number of inflammatory cells was detected at the primary tumour site and in lung metastases (Hobson et al, 2013). This finding indicates the presence of a possible inflammatory reaction after tumour biopsies.

Surgical interventions lead to a wound-healing response accompanied by a number of immunological reactions that have recently been elucidated. The recruitment of inflammatory cells and repolarisation of invading and resident tissue macrophages towards the M2 type is the dominant immunological reaction (Mantovani et al, 2007; Mosser and Edwards, 2008; Mantovani et al, 2013). M1type macrophages are responsible for the elimination of pathogens, tissue destruction and tumour resistance (Mantovani et al, 2007; Sica and Mantovani, 2012; Mantovani et al, 2013). In contrast, M2-type macrophages have immunoregulatory features and are associated with wound-healing, tissue remodelling, angiogenesis and tumour progression (Mantovani et al, 2007; Cao et al, 2011; Hirata et al, 2011; Kurahara et al, 2011; Murray and Wynn, 2011; Hao et al, 2012; Lan et al, 2012; Sica and Mantovani, 2012; Mantovani et al, 2013; Weber et al, 2014). Results from our group showed that increased M2 macrophage polarisation in the epithelial compartment of OSCC patients is correlated with the occurrence of locoregional lymph node metastases (Weber et al, 2014). Furthermore, an increased malignant behaviour of the tumours was associated with a shift towards M2 polarisation in the regional lymph nodes (Wehrhan et al, 2014).

The preoperative diagnostic tumour biopsy-a procedure that is highly relevant for treatment planning-also represents local tissue trauma that can lead to immunological alterations. Therefore, a possible negative influence of biopsies on tumour immunobiology might be associated with a biopsy-induced shift in macrophage polarisation towards the M2 type.

To date, no studies have analysed the possible influence of tumour biopsies on macrophage polarisation in human cancers. Therefore, the aim of this study was to clarify whether macrophage polarisation in diagnostic biopsies of small (pT1 and pT2) primary OSCC differed from that found in definitive tumour resections. To this end, immunohistochemical analyses of the specimens were performed using a computer-assisted quantitative cell counting method.

\section{MATERIALS AND METHODS}

Patients and tissue harvesting. Biopsy and tumour specimens from 34 patients histologically diagnosed with primary OSCC were analysed in this retrospective study. Tumour resection specimens from 34 patients and biopsy specimens from 25 patients were included in this study. The biopsy specimens from nine cases were excluded because they were obtained at other institutions and were not available for analysis or contained only small carcinoma fractions not suitable for analysis. Because we counted macrophage infiltration in at least $1.1 \mathrm{~mm}^{2}$ of carcinoma tissue, specimens with carcinoma fractions smaller than $1 \mathrm{~mm}^{2}$ were considered to be not suitable for analysis and were excluded. All included patients were treated in 2011 at the Department of Oral and Maxillofacial Surgery of the University Hospital Erlangen. The study protocol was approved by the ethical committee of the University of Erlangen-Nuremberg (Ref.-No. 45_12 Bc). The specimens used in this study were obtained from tissue samples collected for routine histopathological diagnosis. Each included specimen was judged by a pathologist to be a representative squamous cell carcinoma. In addition to the main diagnosis of OSCC, the following inclusion criteria were defined: pT1 and pT2 tumours, no restrictions in the grading of the tumour, no adjuvant preoperative radio- or chemotherapy and no organ metastasis at the time of diagnosis.

Only OSCC specimens from patients with pT1 and pT2 were considered because the tumours of these patients were characterised by a better resectability and prognosis compared with the larger pT3 and pT4 tumours (O’Brien et al, 2003). Furthermore, T4 tumours might have a special immunological microenvironment because of their direct contact with bone marrow stem cells (Buenrostro et al, 2014).

Patients with former radio- or chemotherapy and pT3 and pT4 tumours were excluded. No study-related changes in the patients' treatments took place.

The patient cohort $(n=34)$ consisted of 11 patients with a tumour of the tongue, 11 patients with a tumour of the floor of the mouth, 8 with a tumour of the alveolar crest, 3 with a tumour of the palate and 1 with a tumour of the cheek.

The average age of the patients (23 males and 11 females) was 63 years. The pathohistological classified N-status was N0 in 19 cases and $\mathrm{N}+$ in 15 cases. The histological grading was G1 in 2 cases, G2 in 26 cases and G3 in 6 cases. None of the patients in our cohort had distant metastases.

Immunohistochemical staining. The formalin-fixed, paraffinembedded tissue samples were sliced in consecutive, $2-\mu \mathrm{m}$ thick sections with a rotation microtome (Leica, Nussloch, Germany), then dewaxed in xylole and rehydrated in graded propanol before immunohistochemical staining. The immunohistochemical staining was performed with the LSAB (labelled streptavidin-biotin) method and an automated staining device (Autostainer Plus, Dako Cytomation, Hamburg, Germany). The staining kit (Dako Real, Cat. K5001, Dako Cytomation) was used according to the manufactureŕs instructions. Proteins were detected by incubating tissues in the autostainer $\left(21^{\circ} \mathrm{C}, 30 \mathrm{~min}\right)$ with specific antibodies.

The following primary antibodies were used: anti-CD11c (ab52632, clone EP1347y, Abcam, Cambridge, UK), anti-CD68 (11081401, clone KP1, Dako), anti-CD163 (MAB1652, clone K20T, Abnova, Taipei City, Taiwan) and anti-MRC1 (H000043601102, clone 5C11, Abnova).

Biotinylated immunoglobulins were used as the secondary antibody for all samples. DAB + solution (Dako Cytomation) was used as the chromogen. Hematoxylin (Dako Cytomation) was applied for counterstaining of the nuclei. Two consecutive tissue samples were processed per immunohistochemical stain, with one serving as a negative control in each case (identical treatment 
except for replacement of the primary antibody with an IgGisotype of the primary antibody). An appropriate positive control was included in each series.

Quantitative immunohistochemical analysis. The tumour and biopsy sections were completely scanned and digitised using the method of 'whole-slide imaging'. The scanning procedure was performed in cooperation with the Institute of Pathology of the University of Erlangen using a Zeiss MIRAX MIDI Scanner (Zeiss, Jena, Germany). All samples were analysed on a computer (Panoramic MIRAX viewer, Zeiss). Quality controls were performed under a bright-field microscope (Zeiss Axioskop and Axiocam 5, at $\times 100-400$ magnification). H\&E-stained sections of all samples were examined to ensure that all samples contained representative squamous cell carcinoma tissue.

For each sample, three visual fields showing the highest infiltration rate of positive cells were selected (Figure 1) using multi-monitor virtual microscopy (Panoramic MIRAX viewer, Zeiss). The complete area of all three visual fields of one specimen was between 1.1 and $1.5 \mathrm{~mm}^{2}$.

The images showing the visual fields were imported into Biomas (modular systems of applied biology, MSAB, Erlangen, Germany) for cell counting. Two regions of interest were defined in the visual fields using the Biomas software (Erlangen, Germany): the epithelial tumour fraction and the tumour stroma.

A quantitative analysis was performed to determine the numbers of infiltrating CD11c-, CD68-, CD163- and MRC1positive cells in the epithelial tumour fraction and the surrounding stroma. All positive cells in the aforementioned compartments were manually counted. Cell density per $\mathrm{mm}^{2}$ was automatically calculated by the Biomas software. Only cells with a monocytoid/ macrophage-like morphology were counted. Cell counting was performed in a blinded manner by six research fellows familiar with tissue morphology analysis and immunohistochemical methods. In case of a discrepancy, the analyses were discussed with the authors.

Statistical analysis. To analyse the immunohistochemical staining and spatial expression patterns, the cell count per $\mathrm{mm}^{2}$ was determined as the number of positive cells per $\mathrm{mm}^{2}$ of the specimen. Multiple measurements were pooled for each sample group before analysis. The results are expressed as the median, the interquartile range, s.d. and range. Box plot diagrams represent the median, the interquartile range, minimum (Min) and maximum (Max).

Two-sided, adjusted $P$-values $\leqslant 0.05$ were considered to be significant. The analyses were performed using an ANOVA test with SPSS 21 for Mac OS (IBM Inc, New York, NY, USA).

\section{RESULTS}

General morphological considerations. All of the specimens were analysed using a virtual microscope system. In the tumour resection specimens, all analysed markers showed significantly $(P<0.05)$ higher expression in the stroma compared with the epithelial tumour fraction. In the biopsy samples, all stained markers with the exception of CD11c displayed a significantly $(P<0.05)$ higher number of positive cells in the stroma compared with the epithelial tumour compartment.

The stroma of the invasive margin exhibited the highest macrophage infiltration in 12 of the cases (Figure 1). However, the hot spots of macrophage infiltration were randomly distributed in the majority of cases. For each marker, three regions of the highest macrophage infiltration density were selected for cell counting. In eight cases, the preoperative biopsy site was contained by the investigated sections of tumour resection specimens. These areas were excluded from our analyses.

Numerous CD68-positive cells were observed in all analysed cases. A cytoplasmatic expression pattern was found. The stained cells were predominantly round, but some were spindle shaped (Figure 2). In most cases, pale staining of epithelial cells was detectable in addition to distinct staining of the macrophages. This epithelial reactivity included the tumour cells as well as the normal epithelium.

The CD11c-expressing cells were stained both in the cytoplasm and at membrane-bound locations and showed a round shape (Figure 2).

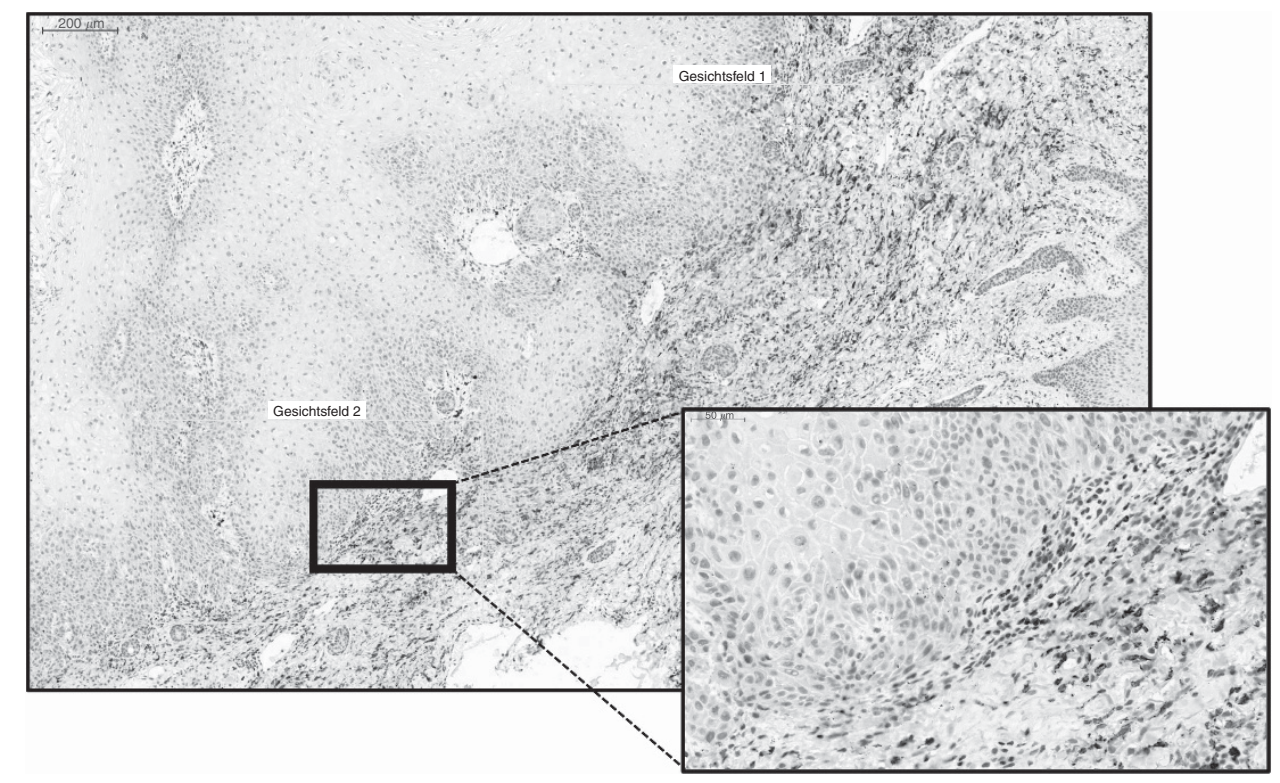

Figure 1. Virtual microscopy and selection of visual fields. The figure shows an exemplary virtual microscope slide. A panoramic view is displayed on the left side and a magnification of the indicated region is displayed on the right side. The magnification can vary continuously and is selected on the computer. Three visual fields containing epithelial tumour fractions and stroma were selected for cell counts. The visual fields are indicated in yellow boxes. The specimen is an oral squamous cell carcinoma biopsy stained for CD163-positive cells. A full colour version of this figure is available online at the British Journal of Cancer website. 
A

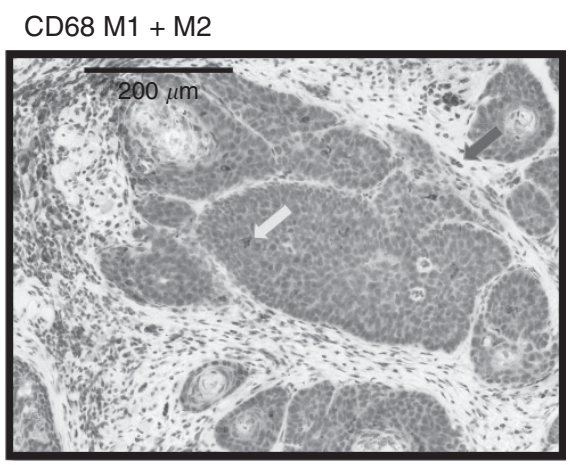

C

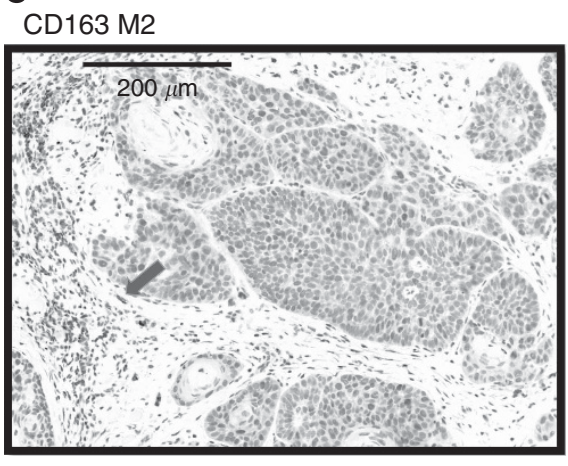

B

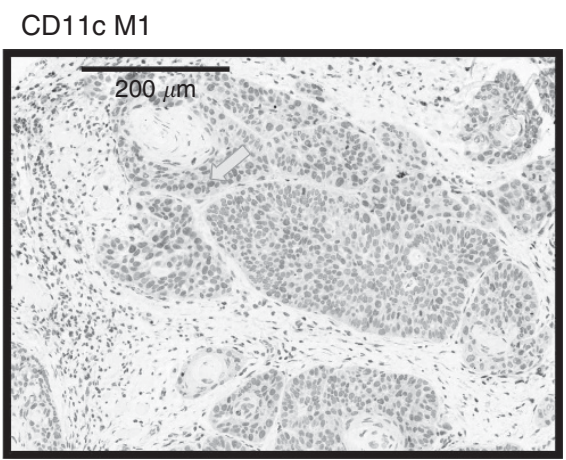

D MRC1 M2

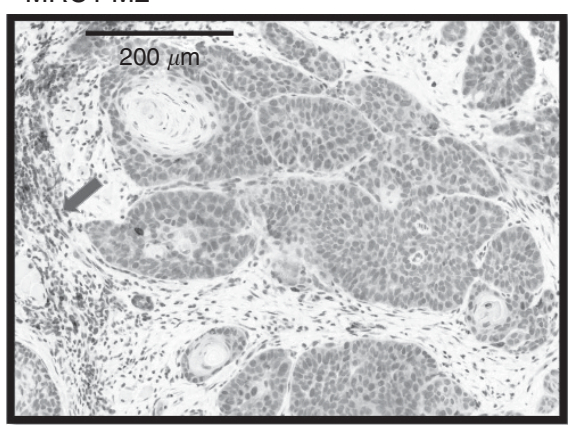

Figure 2. Typical expression patterns of the macrophage markers CD68, CD11c, CD163 and MRC1 in a squamous cell carcinoma biopsy. Exemplary fields of view (original magnification $\times 40$ ) showing the typical expression pattern of the stained macrophage markers CD68, CD11c, CD163 and MRC1 in biopsy specimens. (A) CD68: the marker shows cytoplasmatic staining. The shape of the stained cells includes predominantly round cells (yellow arrow), but some cells also show a spindle shape (red arrow). CD68 is expressed in the epithelial tumour fraction and in the stroma. (B) CD11c: the marker shows a cytoplasmatic expression pattern with an accentuation of the plasma membrane. The shape of the stained cells includes predominantly round cells (yellow arrow). CD11c showed the lowest expression levels of all stained macrophage markers. CD11cexpressing cells were found mainly in the tumour stroma and also in the epithelial tumour fraction. (C) CD163: the marker shows a cytoplasmatic expression pattern with an accentuation of the plasma membrane. The shape of the stained cells includes predominantly spindle-shaped cells (red arrow). CD163 expression was higher in the stroma than in the epithelial tumour compartment. (D) MRC1: the marker shows a cytoplasmatic expression pattern with an accentuation of the plasma membrane. The shape of the stained cells includes predominantly spindle-shaped cells (red arrow). MRC1 expression not only can be found predominantly in the stroma but also in the epithelial tumour fraction. A full colour version of this figure is available online at the British Journal of Cancer website.

Expression of the M2 macrophage marker CD163 was observed in the cytoplasm and bound to the membrane. Most of the stained cells were spindle shaped (Figure 2).

Macrophage marker expression in biopsy and tumour specimens. The mean time between preoperative diagnostic incision biopsy and tumour resection was 15 days (s.d. 9.6) (Table 1), with a range from 2 to 34 days. The time interval between biopsy and tumour resection did not correlate with the difference in macrophage marker expression between biopsies and tumour resections (Table 1).

In the epithelial compartment of tumour specimens, the CD163 cell count (M2 macrophages) was significantly higher (median value of 104 cells per $\mathrm{mm}^{2}$ ) than in the biopsy specimens (median value of 56 cells per $\left.\mathrm{mm}^{2}\right)(P=0.034)$ (Table 2, Figure 3B).

In analogy to the epithelial compartment, CD163 expression in the stroma of the tumour samples (median value of 442 cells per $\mathrm{mm}^{2}$ ) was significantly higher than in the biopsy specimens (median value of 249 cells per $\left.\mathrm{mm}^{2}\right)(P=0.003)$ (Table 2, Figure 3C).

The same relationship was apparent when CD163 expression in the whole analysed specimen area (epithelial + stroma) was taken into consideration. The CD163 expression was significantly higher in the tumour samples $(P=0.00002)$, with a median value of 168 cells per $\mathrm{mm}^{2}$ in biopsy specimens compared with 316 cells per $\mathrm{mm}^{2}$ in tumour samples (Table 2, Figure 3D). The increase in CD163positive cell infiltration was independent of the time between biopsy and tumour resection (Table 1). A total of $92 \%$ of the patients (22 out of 24) showed an increase in CD163-positive cell infiltration in the tumour resection specimens compared with the biopsies. The mean increase in CD163-positive cell infiltration was 108\% (Table 1).

In addition to CD163, CD11c expression in the stroma compartment was significantly higher (median value of 256 cells per $\mathrm{mm}^{2}$ ) in the tumour resection specimens than in the biopsies (median value of 164 cells per $\left.\mathrm{mm}^{2}\right)(P=0.036)$ (Table 2$)$.

There was no significant difference in the expression of other macrophage markers (i.e., CD68 and MRC1) between the tumour and biopsy samples. In summary, the tumour specimens showed significantly increased infiltration of CD163-positive M2 macrophages. This correlation was apparent in all tumour compartments.

Macrophage polarisation in biopsy and tumour specimens. The ratio between CD163-positive cells (predominantly M2 macrophages) and CD68-positive cells (all macrophages) can be considered as the degree of M2 polarisation. In the epithelial compartment, the CD163/CD68 ratio was significantly higher in tumour samples (median value 0.40 ) than in biopsies (median value 0.29$)(P=0.019)$ (Table 3 , Figure $4 \mathrm{~A})$. 
Table 1. Time interval between biopsy and tumour resection and increase im macrophage marker expression

\begin{tabular}{|c|c|c|c|c|c|}
\hline Patient & $\begin{array}{l}\text { Time between biopsy and tumour } \\
\text { resection (in days) }\end{array}$ & $\begin{array}{l}\text { Increase of CD68 } \\
\text { infiltration }\end{array}$ & $\begin{array}{l}\text { Increase of CD11c } \\
\text { infiltration }\end{array}$ & $\begin{array}{l}\text { Increase of CD163 } \\
\text { infiltration }\end{array}$ & $\begin{array}{l}\text { Increase of MRC1 } \\
\text { infiltration }\end{array}$ \\
\hline 1 & 14 & $43 \%$ & $391 \%$ & $141 \%$ & $100 \%$ \\
\hline 2 & 6 & $-37 \%$ & $-50 \%$ & $73 \%$ & $-41 \%$ \\
\hline 3 & 12 & $23 \%$ & $105 \%$ & $243 \%$ & $244 \%$ \\
\hline 4 & 26 & n.a. & n.a. & n.a. & n.a. \\
\hline 5 & 2 & $56 \%$ & $-91 \%$ & $245 \%$ & $55 \%$ \\
\hline 6 & 5 & $-24 \%$ & $-63 \%$ & $35 \%$ & $-9 \%$ \\
\hline 7 & 12 & $46 \%$ & $-43 \%$ & $48 \%$ & $38 \%$ \\
\hline 8 & 18 & $-17 \%$ & $-40 \%$ & $62 \%$ & $18 \%$ \\
\hline 9 & 12 & $86 \%$ & $-37 \%$ & $61 \%$ & $30 \%$ \\
\hline 10 & 8 & $23 \%$ & $154 \%$ & $80 \%$ & $44 \%$ \\
\hline 11 & 12 & $-4 \%$ & $-12 \%$ & $22 \%$ & $-13 \%$ \\
\hline 12 & 32 & $76 \%$ & $18 \%$ & $260 \%$ & $104 \%$ \\
\hline 13 & 6 & $31 \%$ & $32 \%$ & $112 \%$ & $-3 \%$ \\
\hline 14 & 6 & $-17 \%$ & $-62 \%$ & $39 \%$ & $31 \%$ \\
\hline 15 & 6 & $-14 \%$ & $75 \%$ & $19 \%$ & $13 \%$ \\
\hline 16 & 32 & $50 \%$ & $-27 \%$ & $20 \%$ & $-43 \%$ \\
\hline 17 & 33 & $51 \%$ & $24 \%$ & $18 \%$ & $91 \%$ \\
\hline 18 & 34 & $-13 \%$ & $54 \%$ & $29 \%$ & $-26 \%$ \\
\hline 19 & 13 & $-65 \%$ & $1183 \%$ & $-100 \%$ & $104 \%$ \\
\hline 20 & 13 & $3 \%$ & $101 \%$ & $244 \%$ & $20 \%$ \\
\hline 21 & 14 & $58 \%$ & $56 \%$ & $621 \%$ & $277 \%$ \\
\hline 22 & 11 & $-9 \%$ & $87 \%$ & $-26 \%$ & $-38 \%$ \\
\hline 23 & 5 & $37 \%$ & $43 \%$ & $63 \%$ & $-22 \%$ \\
\hline 24 & 16 & $168 \%$ & $-5 \%$ & $128 \%$ & $14 \%$ \\
\hline 25 & 18 & $77 \%$ & $181 \%$ & $150 \%$ & $106 \%$ \\
\hline Mean & 15 & $26 \%$ & $86 \%$ & $108 \%$ & $45 \%$ \\
\hline$P$-value & & 0.264 & 0.972 & 0.948 & 0.713 \\
\hline
\end{tabular}

\section{Table 2. Macrophage marker expression (cells per $\mathrm{mm}^{2}$ ) in biopsy and tumour specimens}

\begin{tabular}{|c|c|c|c|c|c|c|c|c|c|}
\hline \multicolumn{2}{|l|}{ Marker } & \multicolumn{2}{|c|}{ CD68 } & \multicolumn{2}{|c|}{ CD11c } & \multicolumn{2}{|c|}{ CD163 } & \multicolumn{2}{|c|}{ MRC1 } \\
\hline & & Median & s.d. & Median & s.d. & Median & s.d. & Median & s.d. \\
\hline Epithelial fraction & $n$ & & & & & & & & \\
\hline Biopsy & 25 & 306 & 151 & 114 & 143 & 56 & 73 & 154 & 152 \\
\hline Tumour & 34 & 266 & 176 & 148 & 134 & 104 & 119 & 179 & 144 \\
\hline$P$-value & & \multicolumn{2}{|c|}{0.550} & \multicolumn{2}{|c|}{0.949} & \multicolumn{2}{|c|}{0.034} & \multicolumn{2}{|c|}{0.818} \\
\hline Stroma fraction & $n$ & & & & & & & & \\
\hline Biopsy & 25 & 464 & 306 & 164 & 144 & 249 & 211 & 515 & 366 \\
\hline Tumour & 34 & 688 & 287 & 256 & 283 & 442 & 232 & 610 & 277 \\
\hline$P$-value & & \multicolumn{2}{|c|}{0.070} & \multicolumn{2}{|c|}{0.036} & \multicolumn{2}{|c|}{0.003} & \multicolumn{2}{|c|}{0.102} \\
\hline Epithelial + stroma & $n$ & & & & & & & & \\
\hline Biopsy & 25 & 372 & 160 & 168 & 111 & 168 & 92 & 342 & 204 \\
\hline Tumour & 34 & 486 & 164 & 206 & 189 & 316 & 137 & 387 & 177 \\
\hline$P$-value & & \multicolumn{2}{|c|}{0.098} & \multicolumn{2}{|c|}{0.087} & \multicolumn{2}{|c|}{0.000} & \multicolumn{2}{|c|}{0.144} \\
\hline
\end{tabular}


A
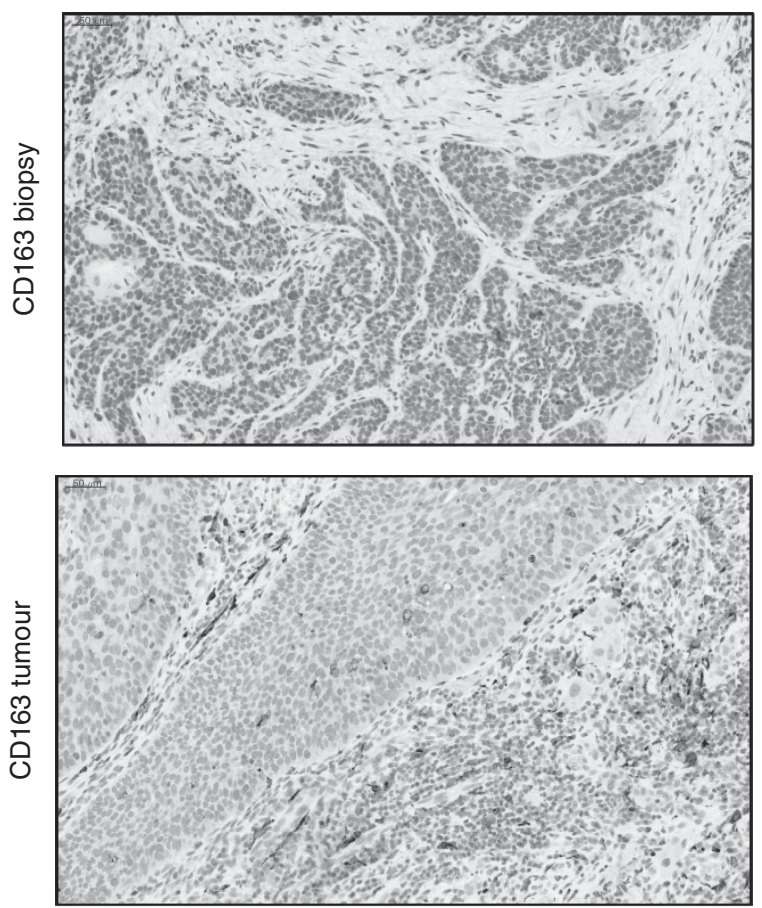

C

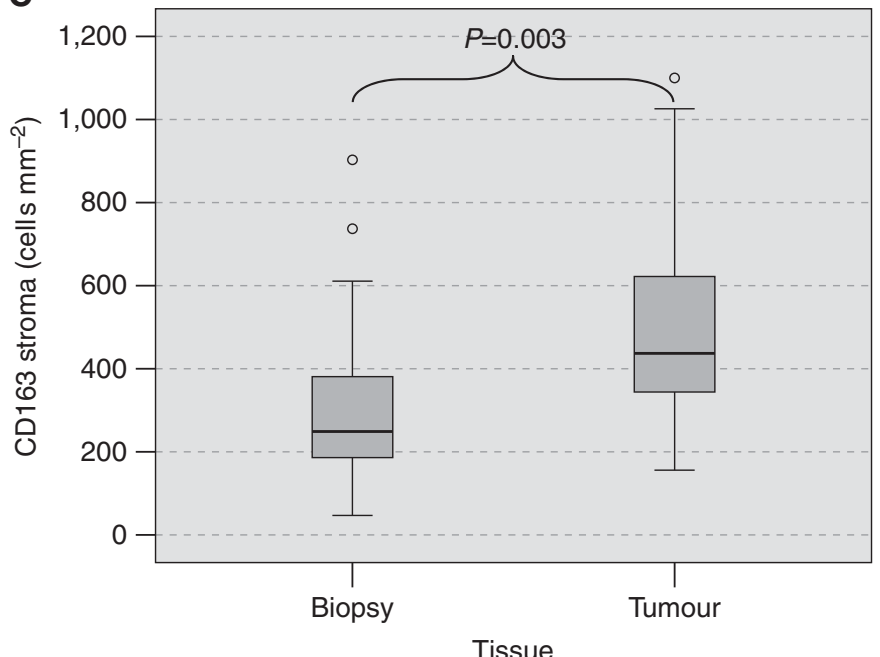

B

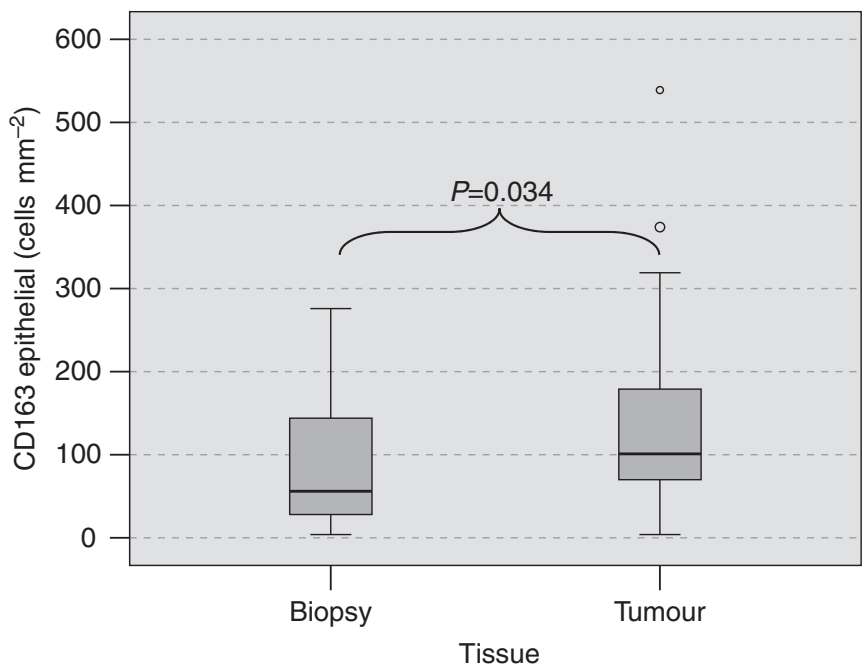

D

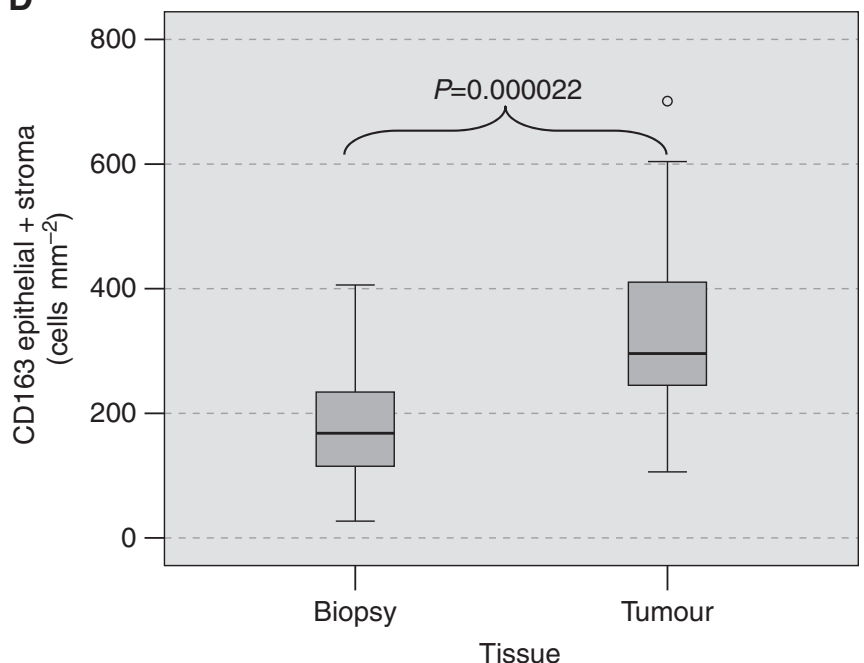

Figure 3. Macrophage cell counts (cells per $\mathrm{mm}^{2}$ ) in biopsy and tumour resection specimens of oral squamous cell carcinomas. (A) The figure shows the expression of the M2 macrophage marker CD163 in biopsy and tumour resection samples from the same patient. The number of CD163expressing cells in the tumour samples is significantly higher in the epithelial tumour compartment and the stroma. (B) The figure shows the median CD163 cell count (cells per $\mathrm{mm}^{2}$ specimen area) in the epithelial compartment of the biopsy and tumour specimens. $P$-values generated by the ANOVA test are indicated. A significantly increased count of CD163-positive cells can be found in the epithelial fraction of the tumour specimens. (C) The figure shows the median CD163 cell count (cells per $\mathrm{mm}^{2}$ specimen area) in the stroma compartment of the biopsy and tumour specimens. $P$-values generated by the ANOVA test are indicated. A significantly increased count of CD163-positive cells can be found in the stroma of tumour specimens. (D) The figure shows the median CD163 cell count (cells per $\mathrm{mm}^{2}$ specimen area) in the whole analysed area (epithelial + stroma) of the biopsy and tumour specimens. P-values generated by the ANOVA test are indicated. A significantly increased count of CD163-positive cells can be found in tumour specimens.

A similar proportion was revealed when the whole analysed area (epithelial + stroma) was taken into consideration. There was a significantly higher ratio in tumour samples (median value 0.64 ) than in biopsy specimens (median value 0.48$)(P=0.003)$ (Table 3 , Figure 4B).

The ratios of other parameters (such as the CD11c/CD68 ratio) did not differ between biopsy and tumour specimens. In summary, tumour specimens showed a significant increase in M2 polarisation.

\section{DISCUSSION}

Value of macrophage polarisation markers. Although the biological relevance of macrophage polarisation in tumour progression is undoubted, an accurate characterisation of the available markers is lacking. The detection of M1 and M2 polarised macrophages using the currently available markers remains problematic (Barros et al, 2013; Wehrhan et al, 2014). For this 
Table 3. Ratio of macrophage marker expression in biopsy and tumour tissue

\begin{tabular}{|c|c|c|c|c|c|c|c|}
\hline & & \multicolumn{2}{|c|}{ CD163/CD68 epithelial } & \multicolumn{2}{|c|}{ CD163/CD68 stroma } & \multicolumn{2}{|c|}{ CD163/CD68 epithelial + stroma } \\
\hline Ratio & & Median & s.d. & Median & s.d. & Median & s.d. \\
\hline Tissue & $n$ & & & & & & \\
\hline Biopsy & 25 & 0.29 & 0.25 & 0.54 & 0.50 & 0.48 & 0.23 \\
\hline Tumour & 34 & 0.40 & 0.25 & 0.74 & 0.30 & 0.64 & 0.27 \\
\hline \multirow[t]{2}{*}{$P$-value } & & \multicolumn{2}{|c|}{0.019} & \multicolumn{2}{|c|}{0.509} & \multicolumn{2}{|c|}{0.003} \\
\hline & & \multicolumn{2}{|c|}{ CD11c/CD68 epithelial } & \multicolumn{2}{|c|}{ CD11c/CD68 stroma } & \multicolumn{2}{|c|}{ CD11c/CD68 epithelial + stroma } \\
\hline Ratio & & Median & s.d. & Median & s.d. & Median & s.d. \\
\hline Tissue & $n$ & & & & & & \\
\hline Biopsy & 25 & 0.47 & 0.43 & 0.37 & 0.26 & 0.45 & 0.28 \\
\hline Tumour & 34 & 0.53 & 0.28 & 0.36 & 0.48 & 0.38 & 0.38 \\
\hline$P$-value & & \multicolumn{2}{|c|}{0.632} & \multicolumn{2}{|c|}{0.236} & \multicolumn{2}{|c|}{0.349} \\
\hline
\end{tabular}

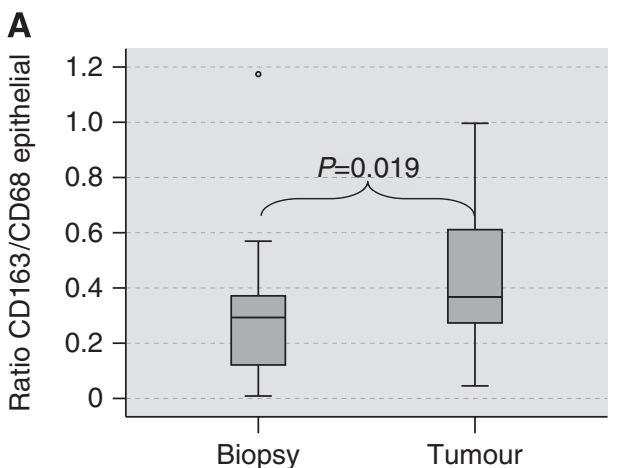

Tissue

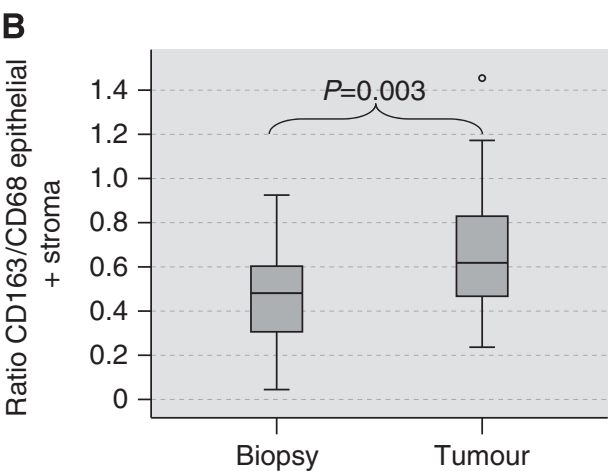

Tissue

Figure 4. Macrophage polarisation in biopsy and tumour resection specimens of oral squamous cell carcinomas. (A) The figure shows the ratio between the CD163 cell count and the CD68 cell count in the epithelial compartment of biopsy and tumour specimens as an indicator of M2 polarisation. P-values generated by the ANOVA test are indicated. A significantly increased CD163/CD68 ratio in the tumour samples compared with the biopsy samples is demonstrated. (B) The figure shows the ratio between the CD163 cell count and the CD68 cell count in the whole analysed area (epithelial + stroma) of biopsy and tumour specimens as an indicator of M2 polarisation. P-values generated by the ANOVA test are indicated. A significantly increased CD163/CD68 ratio in the tumour samples compared with the biopsy samples is demonstrated.

reason, we used macrophage markers frequently described in the literature and successfully used in our previous projects (Weber et al, 2014; Wehrhan et al, 2014). CD68 is the best established generic macrophage marker (Kawamura et al, 2009; Lu et al, 2010; Kurahara et al, 2011; Cho et al, 2012). Immune regulatory and tumourpromoting M2 macrophages are commonly identified by staining the CD163 antigen (Aron-Wisnewsky et al, 2009; Kawamura et al, 2009; Cao et al, 2011; Hasan et al, 2012). Some studies also use MRC1 to detect M2 cells (Aron-Wisnewsky et al, 2009; Hirata et al, 2011; van Putten et al, 2013). In contrast, M1 discriminating markers are not as well defined. CD11c staining is most commonly used to visualise human M1 macrophages (Fischer-Posovszky et al, 2011; Hirata et al, 2011; Cho et al, 2012; Pejnovic et al, 2013).

In addition, the relative proportion of the M1 and M2 markers should also be considered. In analogy to other recent studies dealing with macrophage polarisation in human cancer tissues (Lan et al, 2012; Herwig et al, 2013; Ino et al, 2013; Weber et al, 2014), we aimed to analyse the ratios between the stained markers.

Tumour resection specimens show increased M2 polarisation of infiltrating macrophages compared with preoperative biopsies. In the present study, an increase in M2 polarisation was noted in tumour resection specimens from OSCC compared with samples obtained from preoperative incision biopsies.
CD163 is the most commonly used and best established marker for M2 polarised macrophages (Kawamura et al, 2009; Cao et al, 2011; Hasan et al, 2012). Our data showed a significantly increased infiltration of CD163-positive cells in tumour specimens in all analysed tissue compartments (i.e., epithelial fraction, stroma and whole analysed area) (Table 2). The CD163/CD68 ratio was used as an indicator of M2 polarisation and was significantly higher in the epithelial fraction and the whole analysed area of the tumour specimens compared with the biopsies (Table 3). These data indicate a change in the macrophage composition between biopsies and tumour resections towards M2 polarisation. M2 polarisation of macrophages in OSCC was previously shown to be associated with metastatic growth (Weber et al, 2014) and an unfavourable prognosis (Balermpas et al, 2014).

Expression of CD68, MRC1 and CD11c. The expression of the generic macrophage marker CD68 did not significantly differ between the biopsy and the tumour resection groups. However, a trend towards increased macrophage infiltration in the tumour resection specimens was observed in the stroma compartment (Table 2).

The expression of MRC1 did not differ significantly between tumours and biopsies.

In contrast, significantly higher CD11c expression was detected in the stroma fraction of tumour specimens (Table 2). The absence 
of an established marker for M1 polarised macrophages is an unsolved problem in macrophage research. Several studies have suggested $\mathrm{CD} 11 \mathrm{c}$ as an M1 macrophage marker (FischerPosovszky et al, 2011; Hirata et al, 2011; Cho et al, 2012). However, some authors have also used CD11c as a marker for dendritic cells (Anzai et al, 2012). Thus, one fraction of the CD11cpositive cells might represent dendritic cell subsets. One group analysed the gene expression profile of CD11c low and CD11c high macrophages in decidual tissue (Houser et al, 2011) and detected differential gene expression profiles in both subtypes. Interestingly, both cell types expressed pro- and anti-inflammatory cytokines. Therefore, the authors concluded that CD11c-expressing macrophages did not fit into the classical M1-M2 allocation (Houser et al, 2011). Based on the available data, we can conclude that CD11c cannot be considered a specific marker for M1 polarised macrophages. These data also indicated that macrophage polarisation was more complex than a linear spectrum with M1 and M2 representing the extremes. The lack of a reliable M1 marker is a common problem in macrophage research that still needs to be resolved.

M2 polarisation and the risk of lymph node metastases. Our previous work showed an association between M2 polarised macrophages in the epithelial fraction of OSCC tissue and the occurrence of lymph node metastases (Weber et al, 2014). The tumour-promoting capabilities of M2 macrophages have been demonstrated in vitro and in vivo (Biswas and Mantovani, 2010; Sica and Mantovani, 2012; De Palma and Lewis, 2013; Balermpas et al, 2014).

Recent animal studies revealed a correlation between tumour biopsies and an increased local progression and occurrence of metastases (Hobson et al, 2013). The authors considered immunological factors to be responsible for this phenomenon and detected an increased infiltration of CD45-positive leukocytes at the biopsy site. However, macrophage polarisation was not examined (Hobson et al, 2013). The recent finding that preoperative oral surgery procedures were associated with a worse prognosis and increased risk of developing lymph node metastases in OSCC patients showed that preoperative local tissue trauma influenced tumour biology (Takahashi et al, 2013). Because the preoperative incision biopsy represents a tissue trauma, a tumour biological effect is to be expected.

Because the local tumour microenvironment was shown to influence the polarisation of TAM, the wound-healing reaction consecutive to tissue trauma might serve as a microenvironmental stimulus that affects macrophage polarisation (Kumar and Gabrilovich, 2014).

The presented findings indicate a biopsy-associated shift in macrophage polarisation towards M2. The increased M2 polarisation seems to be independent of the time interval between the biopsy and tumour resection.

Possible mechanisms underlying the biopsy-associated M2 polarisation of macrophages. Biopsy-induced tissue trauma-as with any other surgical procedure-triggers an acute inflammatory reaction and initiates wound-healing processes (Hobson et al, 2013). Initially, tissue damage leads to an acute inflammatory response dominated by M1 macrophages. Later, a shift in macrophage polarisation towards M2 can be observed (Mantovani et al, 2013). M2 macrophages contribute to tumour progression by secreting pro-angiogenic factors such as vascular endothelial growth factor and extracellular matrix remodelling proteins such as matrix metalloproteases (Mantovani et al, 2013). In addition, they induce $\mathrm{T}$-cell tolerance by reducing MHCII expression and the secretion of the immunosuppressive cytokines IL-4, IL-10 and TGF- $\beta$ (Mantovani et al, 2013).

In the case of a two-step surgical procedure with a long time interval between the biopsy and a definitive tumour resection, a shift towards healing associated with M2 polarisation might negatively influence tumour biology.

Limitations of the study. The patient collective (34 tumour resection specimens and 25 biopsy specimens) of this retrospective study was relatively small. No correlation between the duration of the time interval between the biopsy and tumour resection and the increase of M2 polarised macrophages was seen in this study. A larger patient collective could eventually reveal such correlations.

Potential strategies to prevent M2 polarisation in solid tumours. One therapeutic approach targeting possible biopsyinduced M2 polarisation could be the preoperative application of bisphosphonates. The rationale behind this concept is the recently discovered capability of bisphosphonates to repolarise macrophages from a tumour-promoting M2 phenotype to an antitumoural M1 phenotype (Rogers and Holen, 2011).

In contrast to high-dose radiotherapy, low-dose irradiation can shift macrophage polarisation towards the anti-tumoural M1 type (Mantovani et al, 2014). Indeed, 2 Gy of irradiation was able to increase M1 polarisation followed by $\mathrm{T}$-cell recruitment in a tumour xenotransplant mouse model (Klug et al, 2013). Furthermore, irradiation might be capable of inducing an immunostimulatory form of tumour cell death, leading to maturation and activation of antigen presenting cells such as macrophages and dendritic cells (Kulzer et al, 2014; Rubner et al, 2014).

The recently described ability of non-steroidal anti-inflammatory drugs to prevent M2 polarisation ( $\mathrm{Na}$ et al, 2013) has potential therapeutic relevance. Treatment with the non-steroidal antiinflammatory drugs ibuprofen before and after biopsy significantly reduced the number of biopsy-associated lung metastases in mice (Hobson et al, 2013).

\section{CONCLUSION}

We propose that preoperative tumour biopsies lead to increased M2 polarisation of macrophages. This polarisation may be associated with accelerated tumour progression in OSCC.

Prospective studies investigating macrophage markers and the prognosis of patients are needed to prove this hypothesis. These studies should aim to determine whether there is a cut-off time point between biopsy and tumour resection at which macrophage polarisation shifts towards the tumour-promoting M2 type.

\section{ACKNOWLEDGEMENTS}

We thank Luitpold Distel for providing the Biomas analysis software and for assistance with the cell counting procedure. We also thank Carol I. Geppert for assistance with the 'whole-slide imaging' and the digitalisation of the samples. We thank Susanne Schoenherr and Elke Diebel for technical assistance. We also thank the dental students/research fellows Katharina Schoeps, Katharina Loika, Victoria Stanuch, Carina Pirner, Isabell Konopka and Katharina Kaul for processing the tissue specimens, operating the immunohistochemistry autostainer apparatus and cell counting. This study was financially supported by the foundation 'ELAN Fonds der Universität Erlangen' (grant to Manuel Weber in 2012).

\section{CONFLICT OF INTEREST}

The authors declare no conflict of interest. 


\section{AUTHOR CONTRIBUTIONS}

MW formulated the hypothesis, applied for grant support (ELANFonds, University of Erlangen-Nürnberg), initiated and conducted the study, interpreted the data and wrote the manuscript. FW formulated the hypothesis, interpreted the data and contributed relevantly to the manuscript. PM performed the immunohistochemical analyses and the whole-slide imaging of the specimens, helped with cell counting and critically reviewed the manuscript. $\mathrm{MB}$ and $\mathrm{KA}$ helped validate the markers, contributed to the discussion and critically reviewed the manuscript. FN and RP contributed to the discussion and critically reviewed the manuscript. All authors read and approved the final manuscript.

\section{REFERENCES}

Anzai A, Anzai T, Nagai S, Maekawa Y, Naito K, Kaneko H, Sugano Y, Takahashi T, Abe H, Mochizuki S, Sano M, Yoshikawa T, Okada Y, Koyasu S, Ogawa S, Fukuda K (2012) Regulatory role of dendritic cells in postinfarction healing and left ventricular remodeling. Circulation 125: 1234-1245.

Aron-Wisnewsky J, Tordjman J, Poitou C, Darakhshan F, Hugol D, Basdevant A, Aissat A, Guerre-Millo M, Clement K (2009) Human adipose tissue macrophages: $\mathrm{m} 1$ and $\mathrm{m} 2$ cell surface markers in subcutaneous and omental depots and after weight loss. J Clin Endocrinol Metab 94: 4619-4623.

Balermpas P, Rodel F, Liberz R, Oppermann J, Wagenblast J, Ghanaati S, Harter PN, Mittelbronn M, Weiss C, Rodel C, Fokas E (2014) Head and neck cancer relapse after chemoradiotherapy correlates with CD163+ macrophages in primary tumour and CD11b + myeloid cells in recurrences. Br J Cancer 111: 1509-1518.

Barros MH, Hauck F, Dreyer JH, Kempkes B, Niedobitek G (2013) Macrophage polarisation: an immunohistochemical approach for identifying M1 and M2 macrophages. PLoS One 8: e80908.

Biswas SK, Mantovani A (2010) Macrophage plasticity and interaction with lymphocyte subsets: cancer as a paradigm. Nat Immunol 11: 889-896.

Bogden AE, Moreau JP, Eden PA (1997) Proliferative response of human and animal tumours to surgical wounding of normal tissues: onset, duration and inhibition. Br J Cancer 75: 1021-1027.

Buenrostro D, Park SI, Sterling JA (2014) Dissecting the role of bone marrow stromal cells on bone metastases. Biomed Res Int 2014: 875305.

Cao X, Shen D, Patel MM, Tuo J, Johnson TM, Olsen TW, Chan CC (2011) Macrophage polarization in the maculae of age-related macular degeneration: a pilot study. Pathol Int 61: 528-535.

Cho KY, Miyoshi H, Kuroda S, Yasuda H, Kamiyama K, Nakagawara J, Takigami M, Kondo T, Atsumi T (2012) The phenotype of infiltrating macrophages influences arteriosclerotic plaque vulnerability in the carotid artery. J Stroke Cerebrovasc Dis 22(7): 910-918.

De Palma M, Lewis CE (2013) Macrophage regulation of tumor responses to anticancer therapies. Cancer Cell 23: 277-286.

Fischer-Posovszky P, Wang QA, Asterholm IW, Rutkowski JM, Scherer PE (2011) Targeted deletion of adipocytes by apoptosis leads to adipose tissue recruitment of alternatively activated M2 macrophages. Endocrinology 152: 3074-3081.

Hao NB, Lu MH, Fan YH, Cao YL, Zhang ZR, Yang SM (2012) Macrophages in tumor microenvironments and the progression of tumors. Clin Dev Immunol 2012: 948098.

Hasan D, Chalouhi N, Jabbour P, Hashimoto T (2012) Macrophage imbalance (M1 vs. M2) and upregulation of mast cells in wall of ruptured human cerebral aneurysms: preliminary results. J Neuroinflammation 9: 222.

Herwig MC, Bergstrom C, Wells JR, Holler T, Grossniklaus HE (2013) $\mathrm{M} 2 / \mathrm{M} 1$ ratio of tumor associated macrophages and PPAR-gamma expression in uveal melanomas with class 1 and class 2 molecular profiles. Exp Eye Res 107: 52-58.

Hirata Y, Tabata M, Kurobe H, Motoki T, Akaike M, Nishio C, Higashida M, Mikasa H, Nakaya Y, Takanashi S, Igarashi T, Kitagawa T, Sata M (2011) Coronary atherosclerosis is associated with macrophage polarization in epicardial adipose tissue. J Am Coll Cardiol 58: 248-255.

Hobson J, Gummadidala P, Silverstrim B, Grier D, Bunn J, James T, Rincon M (2013) Acute inflammation induced by the biopsy of mouse mammary tumors promotes the development of metastasis. Breast Cancer Res Treat 139: 391-401.
Houser BL, Tilburgs T, Hill J, Nicotra ML, Strominger JL (2011) Two unique human decidual macrophage populations. J Immunol 186: 2633-2642.

Ino Y, Yamazaki-Itoh R, Shimada K, Iwasaki M, Kosuge T, Kanai Y, Hiraoka N (2013) Immune cell infiltration as an indicator of the immune microenvironment of pancreatic cancer. Br J Cancer 108: 914-923.

Kaatsch P, Spix C, Hentschel S, Katalinic A, Luttmann S, Stegmaier C (2013) Krebs in Deutschland 2009/2010. Robert Koch-Institut (Hrsg) und die Gesellschaft der epidemiologischen Krebsregister in Deutschland e.V. (Hrsg). Berlin, Germany.

Kawamura K, Komohara Y, Takaishi K, Katabuchi H, Takeya M (2009) Detection of M2 macrophages and colony-stimulating factor 1 expression in serous and mucinous ovarian epithelial tumors. Pathol Int 59: 300-305.

Keller R (1983) Elicitation of macroscopic metastases via surgery: various forms of surgical intervention differ in their induction of metastatic outgrowth. Invasion Metastasis 3: 183-192.

Klug F, Prakash H, Huber PE, Seibel T, Bender N, Halama N, Pfirschke C, Voss RH, Timke C, Umansky L, Klapproth K, Schakel K, Garbi N, Jager D, Weitz J, Schmitz-Winnenthal H, Hammerling GJ, Beckhove P (2013) Low-dose irradiation programs macrophage differentiation to an iNOS $(+) / \mathrm{M} 1$ phenotype that orchestrates effective T cell immunotherapy. Cancer Cell 24: 589-602.

Krokowski EH (1979) Is the current treatment of Cancer self-limiting in the extent of its success? J Int Acad Prevent Med: 23-39.

Kulzer L, Rubner Y, Deloch L, Allgauer A, Frey B, Fietkau R, Dorrie J, Schaft N, Gaipl US (2014) Norm- and hypo-fractionated radiotherapy is capable of activating human dendritic cells. J Immunotoxicol 11: 328-336.

Kumar V, Gabrilovich DI (2014) Hypoxia inducible factors in regulation of immune responses in tumor microenvironment. Immunology 143(4): 512-519.

Kurahara H, Shinchi H, Mataki Y, Maemura K, Noma H, Kubo F, Sakoda M, Ueno S, Natsugoe S, Takao S (2011) Significance of M2-polarized tumorassociated macrophage in pancreatic cancer. J Surg Res 167: e211-e219.

Lan C, Huang X, Lin S, Huang H, Cai Q, Wan T, Lu J, Liu J (2012) Expression of M2-polarized macrophages is associated with poor prognosis for advanced epithelial ovarian cancer. Technol Cancer Res Treat 12(3): 259-267.

Lange PH, Hekmat K, Bosl G, Kennedy BJ, Fraley EE (1980) Acclerated growth of testicular cancer after cytoreductive surgery. Cancer 45: 1498-1506.

Lu CF, Huang CS, Tjiu JW, Chiang CP (2010) Infiltrating macrophage count: a significant predictor for the progression and prognosis of oral squamous cell carcinomas in Taiwan. Head Neck 32: 18-25.

Mantovani A, Biswas SK, Galdiero MR, Sica A, Locati M (2013) Macrophage plasticity and polarization in tissue repair and remodelling. J Pathol 229: 176-185.

Mantovani A, Sica A, Locati M (2007) New vistas on macrophage differentiation and activation. Eur J Immunol 37: 14-16.

Mantovani A, Vecchi A, Allavena P (2014) Pharmacological modulation of monocytes and macrophages. Curr Opin Pharmacol 17C: 38-44.

Mosser DM, Edwards JP (2008) Exploring the full spectrum of macrophage activation. Nat Rev Immunol 8: 958-969.

Murray PJ, Wynn TA (2011) Obstacles and opportunities for understanding macrophage polarization. J Leukoc Biol 89: 557-563.

Na YR, Yoon YN, Son DI, Seok SH (2013) Cyclooxygenase-2 inhibition blocks M2 macrophage differentiation and suppresses metastasis in murine breast cancer model. PLoS One 8: e63451.

O'brien CJ, Lauer CS, Fredricks S, Clifford AR, Mcneil EB, Bagia JS, Koulmandas C (2003) Tumor thickness influences prognosis of T1 and T2 oral cavity cancer-but what thickness? Head Neck 25: 937-945.

Ohtake K, Shingaki S, Nakajima T (1990) Effects of incision and irradiation on regional lymph node metastasis in carcinoma of the hamster tongue. Oral Surg Oral Med Oral Pathol 70: 62-69.

Pejnovic N, Pantic J, Jovanovic I, Radosavljevic G, Milovanovic M, Nikolic I, Zdravkovic N, Djukic A, Arsenijevic N, Lukic M (2013) Galectin-3 deficiency accelerates high-fat diet induced obesity and amplifies inflammation in adipose tissue and pancreatic islets. Diabetes 62(6): 1932-1944.

Pignon JP, Le Maitre A, Maillard E, Bourhis J. GROUP, M-NC (2009) Meta-analysis of chemotherapy in head and neck cancer (MACH-NC): an update on 93 randomised trials and 17,346 patients. Radiother Oncol 92: 4-14.

Rogers TL, Holen I (2011) Tumour macrophages as potential targets of bisphosphonates. J Transl Med 9: 177.

Rubner Y, Muth C, Strnad A, Derer A, Sieber R, Buslei R, Frey B, Fietkau R, Gaipl US (2014) Fractionated radiotherapy is the main stimulus for the induction of cell death and of Hsp70 release of p53 mutated glioblastoma cell lines. Radiat Oncol 9: 89. 
Sica A, Mantovani A (2012) Macrophage plasticity and polarization: in vivo veritas. J Clin Invest 122: 787-795.

Suzuki K, Shingaki S, Nomura T, Nakajima T (1998) Oral carcinomas detected after extraction of teeth: a clinical and radiographic analysis of 32 cases with special reference to metastasis and survival. Int J Oral Maxillofac Surg 27: 290-294.

Takahashi H, Umeda M, Takahashi Y, Matsui T, Shigeta T, Minamikawa T, Shibuya Y, Komori T (2013) Influence of preoperative dental procedures on the prognosis of patients with squamous cell carcinoma of the gingiva. Br J Oral Maxillofac Surg 51: 108-112.

van Putten SM, Ploeger DT, Popa ER, Bank RA (2013) Macrophage phenotypes in the collagen-induced foreign body reaction in rats. Acta Biomater 9(5): 6502-6510.

Weber M, Buttner-Herold M, Hyckel P, Moebius P, Distel L, Ries J, Amann K, Neukam FW, Wehrhan F (2014) Small oral squamous cell carcinomas with nodal lymphogenic metastasis show increased infiltration of M2 polarized macrophages-an immunohistochemical analysis. J Craniomaxillofac Surg 42: 1087-1094.
Wehrhan F, Buttner-Herold M, Hyckel P, Moebius P, Preidl R, Distel L, Ries J, Amann K, Schmitt C, Neukam FW, Weber M (2014) Increased malignancy of oral squamous cell carcinomas (oscc) is associated with macrophage polarization in regional lymph nodes - an immunohistochemical study. BMC Cancer 14: 522.

Wolff KD, BF, Beck J, Bikowski K, Böhme P, Budach W, Burkhardt A, Danker H, Eberhardt W, Engers K, Fietkau R, Frerich B, Gauler T, Germann G, Gittler-Hebestreit N, Grötz K, Horch R, Ihrler S, Keilholz U, Lell M, Lübbe A, Mantey W, Nusser-Müller-Busch R, Pistner H, Paradies K, Reichert T, Reinert S, Schliephake H, Schmitter M, Singer S, Westhofen M, Wirz S, Wittlinger M (2012) Diagnostik und Therapie des Mundhöhlenkarzinoms. AWMF Leitlinie (German treatment guidelines), 007-100OL.

This work is published under the standard license to publish agreement. After 12 months the work will become freely available and the license terms will switch to a Creative Commons AttributionNonCommercial-Share Alike 4.0 Unported License 